\title{
Squamoid cyst of pancreatic ducts: A case report
}

\begin{abstract}
Jeong-lk Park
Department of Surgery, Ulsan University Hospital, University of Ulsan, College of Medicine, Ulsan, Korea

The term squamoid cyst of pancreatic ducts (SCOP) has been proposed recently, and it is a very rare benign lesion. We report a case of SCOP in a patient who underwent laparoscopic distal pancreatectomy. A 51-year-old woman presented with a pancreatic cystic lesion $(3.3 \mathrm{~cm})$ as an incidental finding on abdominal ultrasonography. A computed tomography scan showed a well-defined cystic lesion in the body of the pancreas with peripheral nodular calcification. Histology showed a unilocular cyst with a thin, fibrotic wall, and it was surrounded by normal-appearing pancreatic tissue. The lining of the cyst was composed of stratified non-keratinized squamous epithelium without significant nuclear atypia. Immunohistochemistry showed positive nuclear p63 expression in the cyst lining. The final diagnosis of SCOP was established. It is important to distinguish SCOPs from mucinous pancreatic cysts that have malignant potential. Preoperative diagnosis of SCOP is still difficult, and further studies are needed to identify specific preoperative characteristics that can accurately distinguish this lesion. (Ann Hepatobiliary Pancreat Surg 2021;25:293-298)
\end{abstract}

Key Words: Cysts; Pancreas; Pancreatic neoplasms

\section{INTRODUCTION}

Currently, the detection of cystic lesions in the pancreas has increased because of the widespread use of high-resolution diagnostic imaging techniques. Many of these lesions are detected during work-up for other medical conditions, and they may have otherwise remained undetected until only a few years ago. Therefore, cystic lesion of the pancreas represents an important category with a challenging differential diagnosis. ${ }^{1-3}$

In 2007, Othman et al. ${ }^{1}$ reported a new type of squamous-lined pancreatic cyst termed squamoid cyst of pancreatic ducts, which is a unilocular cyst with a simple stratified squamous epithelial lining lacking a granular layer and superficial keratinization. In particular, it is important to distinguish this benign cystic lesion from potentially malignant cystic neoplasms, such as mucinous cystic neoplasms (MCNs) and intraductal papillary mucinous neoplasms (IPMNs). ${ }^{1}$

We present a case of a patient with squamoid cyst of the pancreatic duct (SCOP) with a review of the literature.

\section{CASE}

A 51-year-old woman underwent abdominal ultrasonography for routine check-up in a local clinic; a 3.3 $\mathrm{cm}$ sized cystic tumor was detected in the body of the pancreas. She was referred to our hospital for further evaluation. She had no symptoms related to the lesion, and had no remarkable past medical history. On admission to the hospital, physical examination revealed mild tenderness over the epigastrium. Laboratory findings showed normal range of complete blood count, liver function tests, and serum amylase and lipase levels. Serum carbohydrate antigen 19-9 (CA19-9) and carcinoembryonic antigen (CEA) levels were within the normal range. Dynamic computed tomography of the pancreas showed a welldefined unilocular cyst in the body of the pancreas. The lesion measured $4.0 \mathrm{~cm}$ in maximal diameter and had peripheral nodular calcification. There was no evidence of direct communication with the main pancreatic duct, a definite enhancing solid portion, or visualized septum. The main pancreatic duct was not dilated (Fig. 1).

Received: January 31, 2021; Revised: March 5, 2021; Accepted: March 7, 2021

Comesponding author: Jeong-Ik Park

Department of Surgery, Ulsan University Hospital, University of Ulsan, College of Medicine, 877 Bangeojinsunhwando-ro, dong-gu, Ulsan 44033, Korea

Tel: +82-52-250-7109, Fax: +82-52-250-7350, E-mail: jipark@uuh.ulsan.kr

Copyright (C) 2021 by The Korean Association of Hepato-Biliary-Pancreatic Surgery

This is an Open Access article distributed under the terms of the Creative Commons Attribution Non-Commercial License (http://creativecommons.org/ licenses/by-nc/4.0) which permits unrestricted non-commercial use, distribution, and reproduction in any medium, provided the original work is properly cited. Annals of Hepato-Biliary-Pancreatic Surgery • pISSN: 2508-5778 - eISSN: 2508-5859 
The patient underwent laparoscopic distal pancreatectomy because of clinical concern of mucinous cystadenoma. Her recovery was uneventful, and she was discharged 13 days after surgery.

Macroscopically, the cystic lesion of the pancreas was a well-defined unilocular cystic mass without solid area, measuring $3.8 \times 2.2 \times 1.5 \mathrm{~cm}$, and it contained clear serous fluid. Microscopically, the cystic lesion was a well-circumscribed unilocular cyst without papillary configurations. The cyst had a thin, fibrotic wall, and it was surrounded by normal-appearing pancreatic tissue. No lymphoid or splenic tissue was observed. The lining of the cyst was composed of stratified non-keratinized squamous epithelium without significant nuclear atypia or mitosis. The more superficial cells were oriented parallel to the surface. No mucin production was observed (Fig. 2). On immunohistochemical staining for p63, the lining cells showed nuclear positivity with the exception of superficial cells (Fig. 3). The final diagnosis of SCOP was established.

\section{DISCUSSION}

Othman et al. ${ }^{1}$ first described a new type of cystic pan-
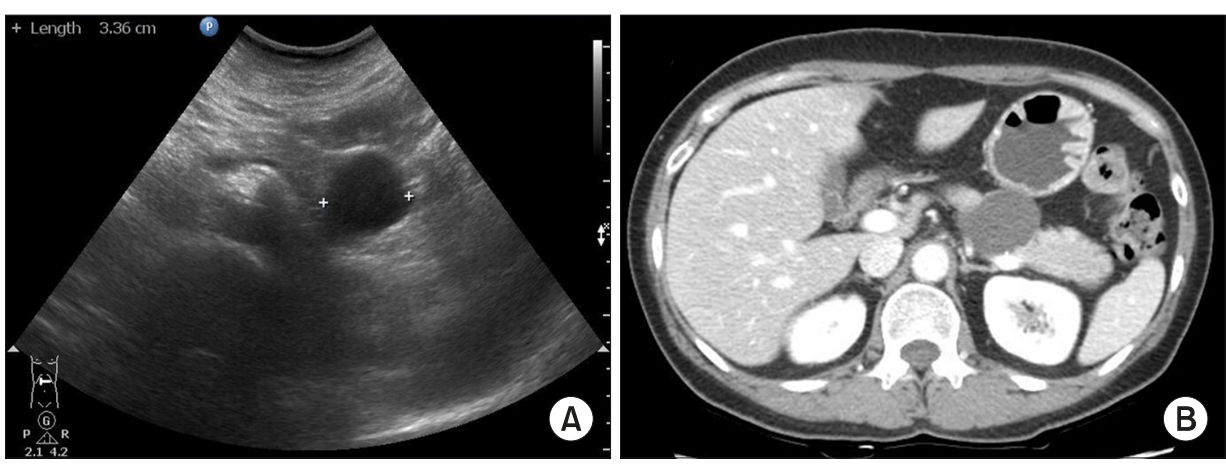

Fig. 1. Abdominal US showed a well-demarcated cyst lesion with a smooth surface (A). CT scan showed a 4-cm sized cystic lesion in the body of the pancreas, with well-defined margin and peripheral nodular calcification (B).
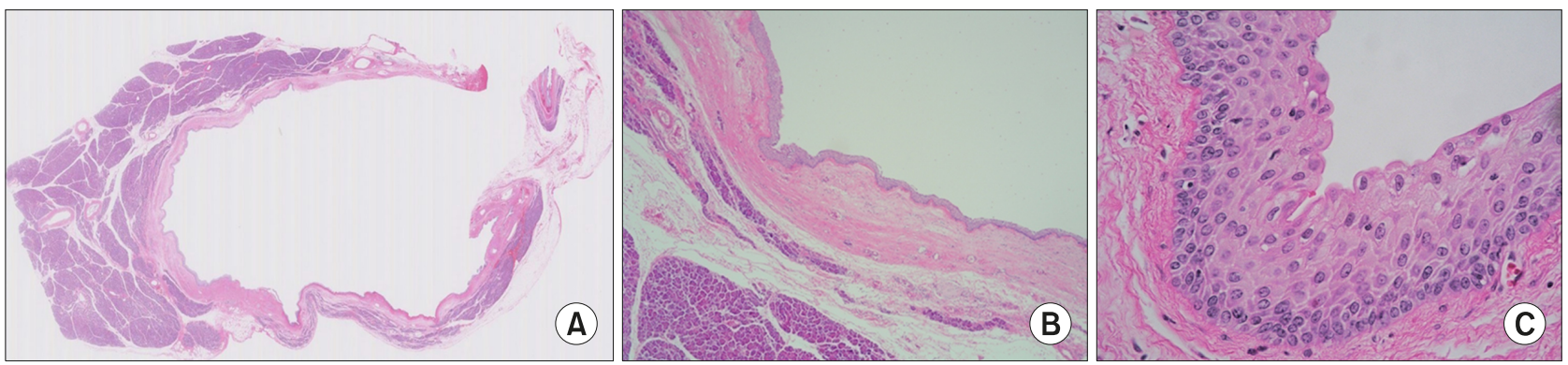

Fig. 2. Unilocular cyst surrounded by pancreatic tissue. The cyst is well-circumscribed with fibrotic wall ((A) Hematoxylin-eosin, $\times 10)$. The cyst lining is composed of stratified squamous epithelium without keratinization ((B) HE $\times 20$; (C) HE $\times 400)$.
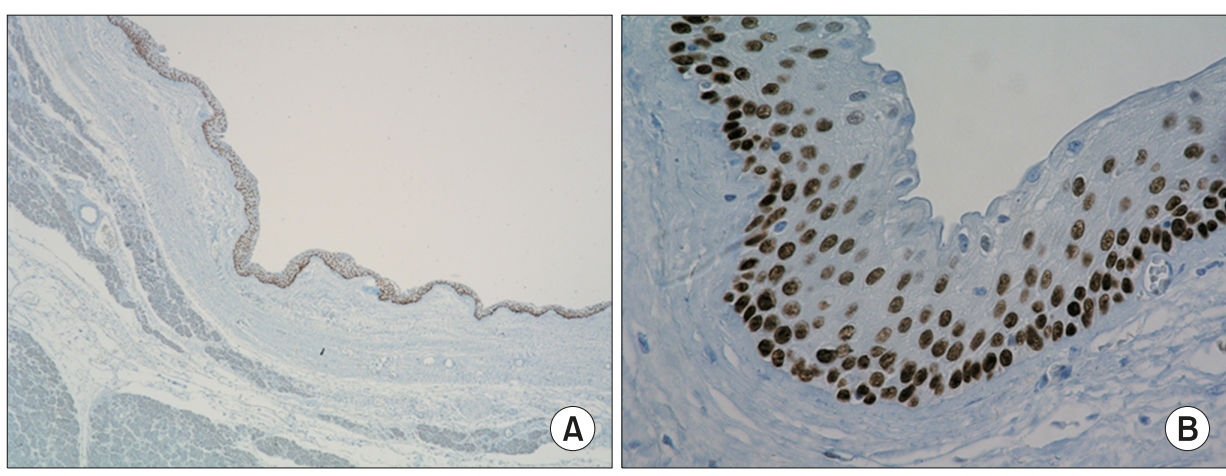

Fig. 3. Immunohistochemstrical staining for p63 shows nuclear staining in cyst lining cells $((\mathrm{A})$ $\times 20$; (B) $\times 400$ ). 
creatic lesion in 2007. It has been called SCOPs, which has morphologic and immunophenotypic characteristics that distinguish it from other cystic lesions in the pancreas. To my knowledge, a total of 33 cases have been reported in the English literature so far, with 16 cases of resection for clinically manifested cysts and 17 cases of incidental identification in resected specimens. ${ }^{1-6}$ The 16 cases of SCOP are summarized in Table 1.

Othman et al. ${ }^{1}$ reported on six cystic lesions, which typically resulted from unilocular cystic dilatation of pancreatic ducts and had variable linings, ranging from attenuated, flat, nonstratified squamous, to transitional, to mucosal-type stratified squamous epithelium (without a cornified layer or parakeratosis). No tall columnar mucinous cells or acinar cells were evident. The wall of the cyst was composed of a thin band of fibrous tissue, with focal islets or tributary ducts, confirming the intraductal nature of the disease process. There was no evidence of pancreatitis (fibrosis or inflammation) in the surrounding tissue. Immunohistochemical analysis demonstrated that MUC1 and MUC6, markers of intercalated duct centroacinar cells, were expressed in the cyst epithelium and the inner layer expressed the nuclear p63, a marker of the squamous/transitional epithelium. GLUT-1, a glycogen clear cell marker positive in most serous tumors, was negative in 2 cases and focally positive in the other 4 cases. Cytokeratin expression profiles were similar to those of pancreatic ducts, showing CK7 and CK19 positivity, but lacking CK20. Ten additional cases of SCOP were identified in pancreatectomy specimens for other causes.

In 2009, Kurahara et al. ${ }^{2}$ reported the first case of SCOP in Japan, a cystic lesion lined by squamous epithelium expressing MUC1, MUC6, and involucrin, a marker of terminal differentiation in stratified squamous epithelia.

Hanson et al. ${ }^{4}$ reported three cases of SCOPs in 2012, and all three cases showed p63, CK7, and CK19 positivity. In addition, the authors discussed about the cyst fluid analysis and cytology. In two cases, preoperative endoscopic ultrasonography guided fine-needle aspiration (EUS guided FNA) and quantitative cyst fluid analysis were performed. The cyst fluid CEA concentrations were $565 \mathrm{ng} / \mathrm{ml}$ and $127 \mathrm{ng} / \mathrm{ml}$ in case 1 and case 3, respectively. In the first report by Othman et al. ${ }^{1}$, the preoperative qualitative cyst fluid chemistry profiles have been re- ported in only 2 cases, with increasing but non-quantified cyst fluid CEA concentrations. The most widely used tumor marker in differentiating non-mucinous from mucinous pancreatic cysts is cyst fluid CEA. A CEA level lower than $30 \mathrm{ng} / \mathrm{ml}$ has a sensitivity of $79 \%$ and a specificity of $73 \%$ for diagnosing a non-mucinous cyst, ${ }^{7}$ while CEA levels greater than $192 \mathrm{ng} / \mathrm{ml}$ have adequate specificity for diagnosing a mucinous cyst. ${ }^{8}$ It is not currently known if this high CEA level can be seen in SCOPs. They suggested that the use of cyst fluid CEA levels to differentiate SCOPs from mucinous cysts was inadequate. Cytological features revealed eosinophilic acellular proteinaceous debris; however, unfortunately these results were not helpful in avoiding misdiagnosis. If exfoliated squamous cells are identified on cytology, it will help to make an accurate diagnosis before performing unnecessary surgery.

Milanetto et al. ${ }^{6}$ reported the first case of SCOP in Italy, a unilocular cyst lined by multilayered squamous epithelium without cytological atypia reacting positively to cytokeratin 7 (CK7, expression profile of pancreatic ducts), but negatively to CK5.

Yoo et $\mathrm{al}^{3}$ reported one case of SCOP, which reacted negatively to MUC1 and MUC6, positively to CK7 and p63, and negatively to inhibin. The authors investigated 5588 pancreatectomy or pancreatic biopsy specimens, and found 7 additional cases of SCOPs. Finally, Assifi et al. ${ }^{5}$ reported four cases of SCOPs.

In these cases, macroscopic and microscopic findings indicated that SCOP represents cystic dilatation of the native duct, rather than de-novo cyst formation; i.e., it is an intraductal process. The immunophenotype confirms the squamous/transitional nature of the epithelial lining (p63 nuclear expression, which is not otherwise detected in any other components of the pancreas), and it also suggests a relationship with the centroacinar/intercalated duct system (with MUC1 and MUC6 expression). ${ }^{1,2}$ In the present case series, immunohistochemical staining was performed and cells expressed p63, and not MUC1 and MUC6. However, Yoo et al. ${ }^{3}$ reported negative expression of MUC1 and MUC6 in their case. Nevertheless, they could determine that SCOP can be diagnosed without the expression of MUC1 or MUC6 because p63 expression in the cells forming the basal region is essential. P63 is a transitional/squamous cell marker, and it is not detected 


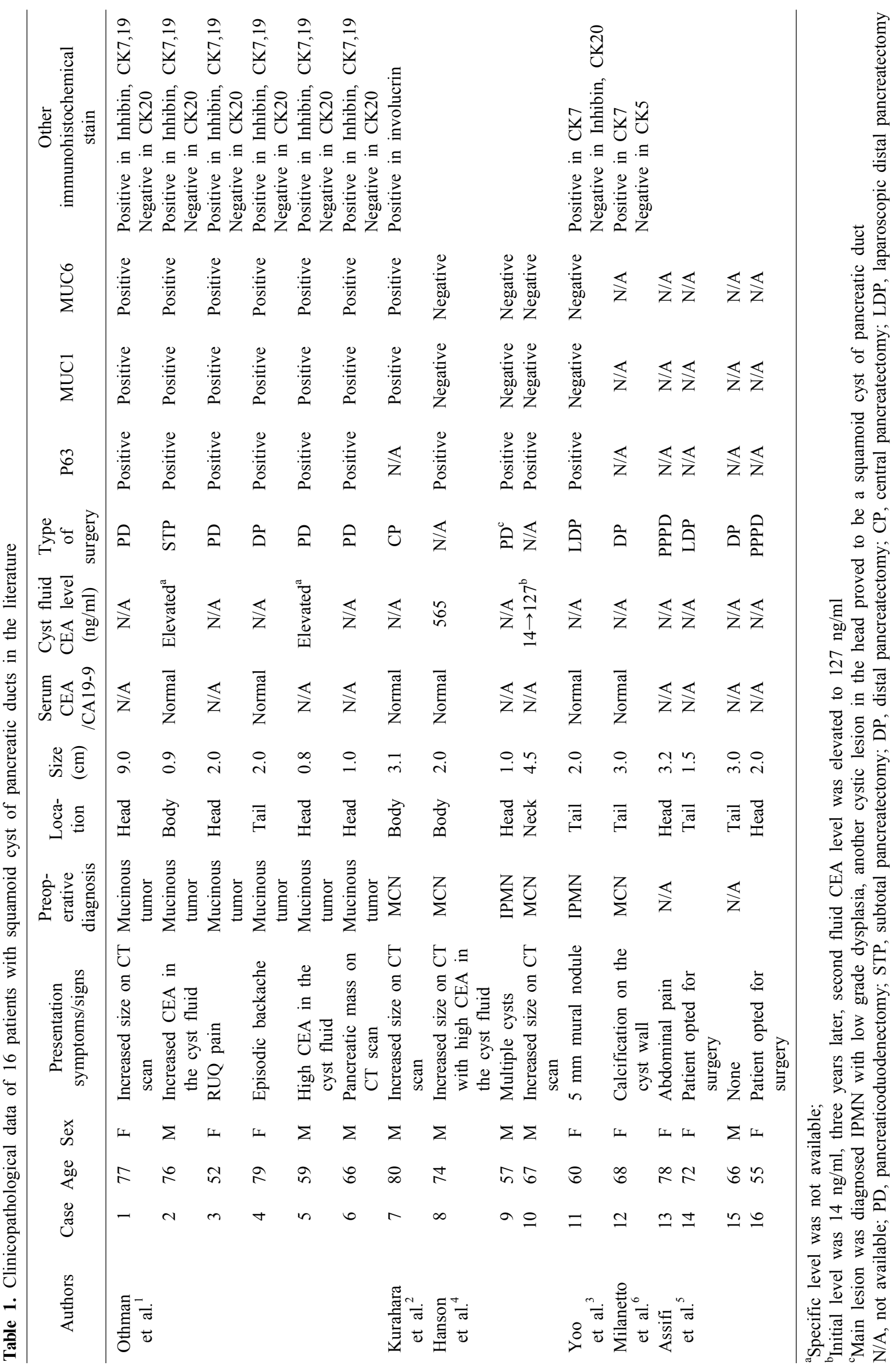


in the normal pancreas or in non-squamous neoplasms.

Squamous cysts of pancreatic ducts need to be distinguished from other types of squamous-lined cysts of the pancreas, such as lymphoepithelial cysts, epidermoid cysts within an intrapancreatic accessory spleen, and dermoid cysts. ${ }^{1}$ Lymphoepithelial cysts may be multilocular or unilocular, and they are lined by well-differentiated stratified squamous epithelium surrounded by a band of mature lymphoid tissue with well-formed germinal centers. ${ }^{1,9}$ Epidermoid cysts within an intrapancreatic accessory spleen are lined by attenuated squamous cells, usually nonstratified, surrounded by normal-appearing splenic tissue. ${ }^{1}$ However, SCOPs lack an underlining lymphoid band or splenic-type tissue characteristic of lymphoepithelial cysts and epidermoid cyst of an intrapancreatic accessory spleen. Most lymphoepithelial cysts are peripancreatic and seem to be intranodal, whereas SCOPs are intraductal and predominantly surrounded by pancreatic tissue. ${ }^{2}$ Parakeratosis is typically seen in lymphoepithelial cysts, which is not a feature of SCOPs. ${ }^{2}$ Dermoid cysts of the pancreas are very rare, and they are morphologically similar to teratomas found in other sites. The presence of adnexal-type elements (sebaceous gland, hair, lymphoid tissue, and even inflammatory cells) is more typical of dermoid cysts; however, these elements are not seen in SCOPs. ${ }^{2,10}$

Cystic dilation of ducts, presumably secondary to downstream obstruction, may occur in the pancreas, and some refer to these entities as retention cysts. SCOPs can also be regarded as a specific type of retention cyst with transitional/squamous transformation; however, an obstructive process is not evident. ${ }^{1}$

All these cystic lesions of the pancreas are thought to be benign. Clinically, it is important to distinguish SCOPs from mucinous cyst-forming neoplasms, such as MCNs or IPMNs. In contrast to SCOPs, these mucinous lesions carry the risk of malignant transformation and have potential to progress to invasive adenocarcinoma. However, the differential diagnosis of pancreatic cystic lesions is difficult because the radiologic and clinical findings are not characteristic. IPMNs are characterized by cystic dilatation of pancreatic ducts in which intraductal proliferation of neoplastic mucin-producing cells is usually arranged in a papillary pattern. MCNs are characterized by an ovarian-type stroma that forms a layer of variable thickness beneath the epithelial lining. These neoplasms are com- posed of large multilocular cysts of size $1 \mathrm{~cm}$ or greater. The cysts have thick fibrous walls, and they do not visibly communicate with the main pancreatic duct. ${ }^{2}$ Like in almost all patients in the reported cases of SCOPs in the literature, the case in this study was also radiologically diagnosed with MCN and it was decided to perform surgical resection.

In conclusion, the term SCOP has been proposed recently, and it is a very rare benign lesion. With the improvement in imaging techniques, SCOPs are being increasingly recognized and should be considered in the differential diagnoses of pancreatic cystic lesions. As shown in the literature, SCOPs are often indistinguishable from cystic neoplasms of the pancreas preoperatively; most cases of SCOPs are confirmed after surgical resection in order to exclude malignancy. Identification of the squamous epithelium in cytological samples through EUS guided FNA may help avoid unnecessary extended resections. SCOP remains a difficult diagnosis to establish preoperatively, and further studies are needed to identify specific preoperative characteristics that can accurately differentiate this lesion.

\section{CONFLICT OF INTEREST}

No potential conflict of interest relevant to this article was reported.

\section{ORCID}

Jeong-Ik Park: https://orcid.org/0000-0002-1986-9246

\section{REFERENCES}

1. Othman M, Basturk O, Groisman G, Krasinskas A, Adsay NV. Squamoid cyst of pancreatic ducts: a distinct type of cystic lesion in the pancreas. Am J Surg Pathol 2007;31:291-297.

2. Kurahara H, Shinchi H, Mataki Y, Maeda S, Takao S. A case of squamoid cyst of pancreatic ducts. Pancreas 2009;38:349-351.

3. Yoo DG, Hwang S, Hwang DW, Kim KH, Ahn CS, Ha TY, et al. Case report of a pancreatic squamoid cyst. Korean J Hepatobiliary Pancreat Surg 2013;17:181-185.

4. Hanson JA, Salem RR, Mitchell KA. Squamoid cyst of pancreatic ducts: a case series describing novel immunohistochemistry, cytology, and quantitative cyst fluid chemistry. Arch Pathol Lab Med 2014;138:270-273.

5. Assifi MM, Nguyen PD, Agrawal N, Dedania N, Kennedy EP, Sauter PK, et al. Non-neoplastic epithelial cysts of the pancreas: a rare, benign entity. J Gastrointest Surg 2014;18:523-531.

6. Milanetto AC, Iaria L, Alaggio R, Pedrazzoli S, Pasquali C. 
Squamoid cyst of pancreatic ducts: a challenging differential diagnosis among benign pancreatic cysts. JOP 201314:657-660.

7. Snozek CL, Mascarenhas RC, O'Kane DJ. Use of cyst fluid CEA, CA19-9, and amylase for evaluation of pancreatic lesions. Clin Biochem 2009;42:1585-1588.

8. Jani N, Bani Hani M, Schulick RD, Hruban RH, Cunningham SC. Diagnosis and management of cystic lesions of the pancreas. Diagn Ther Endosc 2011;2011:478913.
9. Adsay NV, Hasteh F, Cheng JD, Klimstra DS. Squamous-lined cysts of the pancreas: lymphoepithelial cysts, dermoid cysts (teratomas), and accessory-splenic epidermoid cysts. Semin Diagn Pathol 2000;17:56-65.

10. Fernandez-Cebrian JM, Carda P, Morales V, Galindo J. Dermoid cyst of the pancreas: a rare cystic neoplasm. Hepatogastroenterology 1998;45:1874-1876. 